\title{
ALGUNOS ASPECTOS DE LA TEORÍA DE ONDÍCULAS
}

\author{
Luis Miguel Nuñez Ramirez ${ }^{1}$, \\ Humberto Emiliano Galvez Perez ${ }^{2}$ \& Roland Hubert Peña Flores ${ }^{3}$
}

Resumen: El objetivo de este trabajo es analizar brevemente, algunas estrategias que han sido desarrolladas para la construcción del polinomio trigonométrico $m_{0}$ bajo ciertas condiciones. Asimismo, mostraremos la relación que existe entre la función escala $\phi$, la Ondícula $\Psi$ y el polinomio trigonométrico $m_{0}$.

Por ello, el Análisis Multiresolución (AMR) juega un rol vital para la construcción de bases ortonormales de Ondículas. Dentro de las nuevas técnicas usadas, una de las más importantes es la teoría de Frames que tiene una relación directa con el análisis de Ondículas.

Palabras clave: Función escala $\phi$, Ondicula $\Psi$, polinomio trigonométrico $m_{0}$, AMR y Frames.

\section{SOME ASPECTS OF THE THEORY OF WAVELETS}

\begin{abstract}
The aim of this paper is to briefly discuss some strategies that have been developed for the construction of trigonometric polynomial $m_{0}$ under certain conditions. Also show the relationship between the function scale $\phi$, the wavelet and the trigonometric polynomial $m_{0}$.

Therefore, the Multiresolution Analysis (AMR) plays a vital role in the construction of orthonormal bases of wavelets. Among the new techniques used, one of the most important is the theory of frames that have a direct relationship with the wavelets analysis.
\end{abstract}

Key words: Function scale $\phi$, wavelets $\Psi$, trigonometric polynomial $m_{0}$, AMR and Frames.

\section{Introducción}

El análisis de "Ondículas" es una alternativa al clásico análisis de Fourier localizado. Hacemos uso de la tecnica de las "ondículas" dado que el análisis de Fourier resulta insuficiente para interpretar y resolver otras situaciones que provienen, por ejemplo, de la teoría de las señales.

Nuestro objetivo es analizar algunas técnicas para la construcción de los elementos básicos del AMR, como son por ejemplo los espacios $V_{j}^{\prime} s$ o la función escala $\phi$. Asimismo, veremos la relación que existe entre la Ondícula $\Psi$ y el polinomio trigonométrico $m_{0}$.

Los caminos para construir la estructura de un AMR estan dadas por:

(i) Los espacios $V_{j}^{\prime} s$.

(ii) La función escala $\phi$.

${ }^{1}$ UNMSM, Facultad de Ciencias Matemáticas, Lima- Perú, e-mail: lnuñezr@unmsm.edu.pe

${ }^{2}$ UNMSM, Facultad de Ciencias Matemáticas, Lima - Perú, e-mail: hgalvezp@unmsm.edu.pe

${ }^{3}$ UNMSM, Facultad de Ciencias Matemáticas, Lima - Perú, e-mail: rpeñaf@unmsm.edu.pe 
(iii) Los coeficientes $\sqrt{2} h(k)$ de la ecuación "dilatación"

$$
\phi(t)=\sum_{k \in \mathbb{Z}} \sqrt{2} \bar{h}(k) \phi(2 t-k) .
$$

La estrategia a emplear para encontrar la conexión y relación de las propiedades de $\phi$ y $\Psi$ está basada en el siguiente esquema recursivo:

(i) Calcular los coeficientes de la aproximación de la señal $f$, vía:

$$
f_{j}(t)=\sum_{k \in \mathbb{Z}} a_{j k} \phi_{j k}(t) \quad, \quad f(t)=\sum_{k \in \mathbb{Z}} b_{j k} \Psi_{j k}(t)
$$

(ii) Usar el método de la "cascada" para la construcción de $\phi(t)$ resolviendo la ecuación de "dilatación".

(iii) $\operatorname{Si} \bar{c}(k)^{\prime} s \perp \bar{d}(k)^{\prime} s$ entonces $\Psi_{j k}(t) \perp \phi_{j k}$.

Las Ondículas en estos últimos años han logrado un sorprendente y notable desarrollo, siendo una de sus mejores exponentes Ingrid Daubechies [7] quien actualmente esta trabajando en las llamadas Ondículas de segunda generación, esto es, permite la implementación rápida de la transformada de Ondículas ("lifting scheme").

Asimismo Ronald Coifman [5] aplicó las Ondículas para desruidar datos. Su trabajo con Beylkin y Rokhlin [1] sobre ciertos problemas computacionales en análisis numérico permitió resolver problemas de alta concentración computacional y que no había sido posible tratarse con los algoritmos existentes.

El uso más común de las Ondículas se encuentra en sus aplicaciones al procesamiento de señales.

\section{Correspondencia con Bases Ortonormales de Ondículas}

\subsection{Relación entre $\widehat{\phi}$ y $m_{0}$}

Comencemos por encontrar una fórmula para la función escala $\phi$, lo cual es un posible candidato. Realizado esto, procederemos a verificar si esté posible candidato define un buen AMR.

$\mathrm{Si}$ un polinomio trigonométrico $m_{0}$ es asociado con un $\mathrm{AMR}$, y la función escala $\phi \in L^{1}(\mathbb{R})$ entonces sabemos que

$$
\widehat{\phi}(\xi)=m_{0}\left(\frac{\xi}{2}\right) \widehat{\phi}\left(\frac{\xi}{2}\right), \forall \xi
$$

Ademas, si $\widehat{\phi}$ es acotada y continua en 0 , entonces es necesaria la condición $\widehat{\phi}(0) \neq 0$, debido a la densidad de los subespacios $V_{j}$ en $L^{2}(\mathbb{R})$. Luego, en (1.1) cuando $\xi=0$ se tiene que $\widehat{\phi}(0)=m_{0}(0) \widehat{\phi}(0)$, esto es, $m_{0}(0)=1$.

Asimismo, sabemos que

$$
\left|m_{0}(\xi)\right|^{2}+\left|m_{0}(\xi+\pi)\right|^{2}=1, \text { en c.t.p, }
$$

entonces cuando $\xi=0$ y debido a que $m_{0}(0)=1$, en $(2.2)$ tenemos $m_{0}(\pi)=0$.

De (1.1), se obtiene

$$
\widehat{\phi}(\xi)=\prod_{j=1}^{l} m_{0}\left(\frac{\xi}{2^{j}}\right) \widehat{\phi}\left(\frac{\xi}{2^{j}}\right)
$$


De aqui, $\forall k \in \mathbb{Z}-\{0\}$, se tiene que

$$
\widehat{\phi}(2 k \pi)=\widehat{\phi}\left(2.2^{l}(2 m+1) \pi\right) \text { donde } k=2^{l}(2 m+1) \text { para algún } l \geqslant 0, m \in \mathbb{Z} .
$$

Sustituyendo $\xi=2.2^{l}(2 m+1) \pi$ en $(2.3)$, obtenemos

$$
\widehat{\phi}(2 k \pi)=\prod_{j=1}^{l} m_{0}\left(2^{l+1-j}(2 m+1) \pi\right) \widehat{\phi}(2(2 m+1) \pi)
$$

y debido a (1.1) tenemos

$$
\widehat{\phi}(2 k \pi)=\prod_{j=1}^{l} m_{0}\left(2^{l+1-j}(2 m+1) \pi\right) m_{0}\left(\frac{2(2 m+1) \pi}{2}\right) \widehat{\phi}\left(\frac{2(2 m+1) \pi}{2}\right) .
$$

Luego, como $m_{0}$ es una función $2 \pi$-periódica y $m_{0}(\pi)=0$, se tiene que

$$
\widehat{\phi}(2 k \pi)=\prod_{j=1}^{l} m_{0}\left(2^{l+1-j}(2 m+1) \pi\right) m_{0}(\pi) \widehat{\phi}((2 m+1) \pi),
$$

esto es,

$$
\widehat{\phi}(2 k \pi)=m_{0}(\pi) \widehat{\phi}((2 m+1) \pi)=0, \forall \dot{k} \in \mathbb{Z}-\{0\}
$$

Desde que

$$
\sum_{l \in \mathbb{Z}}|\widehat{\phi}(\xi+2 \pi l)|^{2}=(2 \pi)^{-1}, \text { en c.t.p. }
$$

entonces

$$
|\widehat{\phi}(\xi)|^{2}+\sum_{\substack{l \in \mathbb{Z} \\ l \neq 0}}|\widehat{\phi}(\xi+2 \pi l)|^{2}=(2 \pi)^{-1}
$$

y cuando $\xi=0$, se tiene

$$
|\widehat{\phi}(0)|^{2}+\sum_{\substack{l \in \mathbb{Z} \\ l \neq 0}}|\widehat{\phi}(2 \pi l)|^{2}=(2 \pi)^{-1}
$$

pero por (3.5) se tiene que $\widehat{\phi}(2 \pi l)=0, \forall l \in \mathbb{Z}-\{0\}$. Por tanto

$$
|\widehat{\phi}(0)|=(2 \pi)^{-\frac{1}{2}} \text {. }
$$

Ahora, como $\widehat{\phi}(\xi)=\int \exp (-i \xi x) \phi(x) d x$, cuando $\xi=0$ tenemos

$$
\widehat{\phi}(0)=\int \phi(x) d x
$$

entonces es equivalente afirmar que

$$
|\widehat{\phi}(0)|=(2 \pi)^{-\frac{1}{2}} \quad \text { ó }\left|\int \phi(x) d x\right|=1 \text {. }
$$

Esto es conveniente para elegir la forma de $\phi$ de manera que $\int \phi(x) d x=1$.

Por otro lado, se tiene que

$$
\widehat{\phi}(\xi)=\prod_{j=1}^{l} m_{0}\left(\frac{\xi}{2}\right) \widehat{\phi}\left(\frac{\xi}{2^{l}}\right) \longrightarrow \widehat{\phi}(0) \prod_{j=1}^{\infty} m_{0}\left(\frac{\xi}{2^{j}}\right)
$$


converge absolutamente y uniformemente sobre compactos, cuando $l \rightarrow+\infty$. Esto es,

$$
\widehat{\phi}(\xi)=\widehat{\phi}(0) \prod_{j=1}^{\infty} m_{0}\left(\frac{\xi}{2^{j}}\right)=(2 \pi)^{-\frac{1}{2}} \prod_{j=1}^{\infty} m_{0}\left(\frac{\xi}{2^{j}}\right)
$$

y tiene sentido "escribir" este producto infinito: Desde que,

$$
\sum_{n}\left|h_{n}\right||n|<\infty, \quad m_{0}(0)=1, \quad m_{0}(\xi)=2^{-\frac{1}{2}} \sum_{n} h_{n} \exp (-i n \xi),
$$

tenemos que

$$
\left|m_{0}(\xi)\right|=\left|m_{0}(\xi)+1-1\right|=\left|1+\left(m_{0}(\xi)-1\right)\right| \leq 1+\left|m_{0}(\xi)-1\right|
$$

y como $m_{0}(0)=\frac{1}{\sqrt{2}} \sum_{n} h_{n}=1$, se tiene que

$$
\begin{aligned}
\left|m_{0}(\xi)-1\right| & =\left|\frac{1}{\sqrt{2}} \sum_{n} h_{n} \exp (-i n \xi)-\frac{1}{\sqrt{2}} \sum_{n} h_{n}\right| \\
& \leq \frac{1}{\sqrt{2}} \sum_{n}\left|h_{n}\right||\exp (-i n \xi)-1|
\end{aligned}
$$

Pero

$$
|\exp (-i n \xi)-1| \leq|n \xi|
$$

luego haciendo $c=\frac{1}{\sqrt{2}} \sum_{n}\left|h_{n}\right||n|$, obtenemos

$$
\left|m_{0}(\xi)-1\right| \leq c|\xi|
$$

Así

$$
\left|m_{0}(\xi)\right| \leq 1+\left|m_{0}(\xi)-1\right| \leq 1+c|\xi| \leq \exp (c|\xi|)
$$

Por consiguiente

$$
\left|m_{0}\left(2^{-j} \xi\right)\right| \leq \exp \left(c\left|2^{-j \xi}\right|\right)=\exp \left(c 2^{-j|\xi|}\right)
$$

luego

$$
\prod_{j=1}^{l}\left|m_{0}\left(2^{-j} \xi\right)\right| \leq \prod_{j=1}^{l} \exp \left(c 2^{-j}|\xi|\right)=\exp \left(c|\xi| \sum_{j=1}^{l} 2^{-j}\right) .
$$

Por tanto, cuando $j \rightarrow \infty$ concluimos que

$$
\prod_{j=1}^{\infty}\left|m_{0}\left(2^{-j} \xi\right)\right| \leq \exp (c|\xi|)
$$

Observación. Generalmente, todo esto es aplicable, cuando $\phi \in L^{1}(\mathbb{R})$ y los $h_{n}$ tienen suficiente decaimiento. Para nuestro caso, nos interesa construir un $\phi$ de soporte compacto, a partir del polinomio trigonométrico $m_{0}$. Además, sabemos que si $\phi \in L^{2}(\mathbb{R}), \phi$ con soporte compacto, entonces $\phi \in L^{1}(\mathbb{R})$.

Notamos que $\widehat{\phi}(\xi)=(2 \pi)^{-\frac{1}{2}} \prod_{j=1}^{\infty} m_{0}\left(2^{-j} \xi\right)$ es solamente un posible "candidato" para la función escala $\phi$ correspondiente a un polinomio trigonométrico $m_{0}$. Necesitamos que $\phi$, satisfaga algún "requisito" básico para una función escala. 


\section{Los Frames Apretados}

Teorema 1. Sea $m_{0}$ un polinomio trigonométrico que satisface la condición (2.2) y que $m_{0}(0)=1$. Además, consideremos $\phi, \Psi$ funciones de soporte compacto en $L^{2}(\mathbb{R})$ definidas por:

$$
\widehat{\phi}(\xi)=(2 \pi)^{-\frac{1}{2}} \prod_{j=1}^{\infty} m_{0}\left(2^{-j} \xi\right) \quad y \Psi(\xi)=2^{\frac{1}{2}} \sum_{n}(-1)^{n} \bar{h}_{1-n} \phi(2 x-n)
$$

respectivamente. Entonces

$$
\sum_{j, k \in \mathbb{Z}}\left|\left\langle f, \Psi_{j, k}\right\rangle\right|^{2}=\|f\|^{2}, \forall f \in L^{2}(\mathbb{R})
$$

donde $\Psi_{j, k}(x)=2^{-\frac{j}{2}} \Psi\left(2^{-j} x-k\right)$. Es decir, $\left\{\Psi_{j, k}: j, k \in \mathbb{Z}\right\}$ constituye un "Frame Apretado" $\operatorname{para} L^{2}(\mathbb{R})$.

\section{Demostración.}

(a) Sabemos que la condición (2.2) puede ser expresada como

$$
\sum_{m} h_{m} \bar{h}_{m-2 k}=\delta_{k, 0}
$$

(b) Considerando $f \in C_{c}^{\infty}$ ( $f$ de soporte compacto y $\left.C^{\infty}\right)$, se obtiene

$$
\sum_{k}\left|\left\langle f, \phi_{j, k}\right\rangle\right|^{2}=2^{-j} \sum_{k}\left|\int_{\mathbb{R}} f(x) \phi\left(2^{-j} x-k\right) d x\right|^{2} .
$$

Por otra parte

$$
\left|\int_{\mathbb{R}} f(x) \phi\left(2^{-j} x-k\right) d x\right| \leq \int_{\mathbb{R}}|f(x)|\left|\phi\left(2^{-j} x-k\right)\right| d x
$$

entonces

$$
\sum_{k}\left|\left\langle f, \phi_{j, k}\right\rangle\right|^{2} \leq 2^{-j} \sum_{k}\left[\int_{\mathbb{R}}|f(x)|\left|\phi\left(2^{-j} x-k\right)\right| d x\right]^{2}
$$

y debido a que $f \in C_{c}^{\infty}$, tenemos

$$
\sum_{k}\left|\left\langle f, \phi_{j, k}\right\rangle\right|^{2} \leq 2^{-j} \sum_{k}\left[\int_{S o p(f)}\|f\|_{\infty}\left|\phi\left(2^{-j} x-k\right)\right| d x\right]^{2},
$$

es decir,

$$
\sum_{k}\left|\left\langle f, \phi_{j, k}\right\rangle\right|^{2} \leq 2^{-j}\|f\|_{\infty}^{2} \sum_{k}\left[\int_{S o p(f)}\left|\phi\left(2^{-j} x-k\right)\right| d x\right]^{2} .
$$

Luego, aplicando la Desigualdad de Holder se tiene

$$
\sum_{k}\left|\left\langle f, \phi_{j, k}\right\rangle\right|^{2} \leq 2^{-j}\|f\|_{\infty}^{2} \sum_{k}\left[\int_{S o p(f)}\left|\phi\left(2^{-j} x-k\right)\right|^{2} d x\right]\left[\int_{S o p(f)} d x\right]
$$

Entonces

$$
\sum_{k}\left|\left\langle f, \phi_{j, k}\right\rangle\right|^{2} \leq 2^{-j}\|f\|_{\infty}^{2}|S o p(f)|\left[\sum_{k} \int_{S o p(f)}\left|\phi\left(2^{-j} x-k\right)\right|^{2} d x\right]
$$


y haciendo el cambio $x=2^{j} y$, obtenemos

$$
\sum_{k}\left|\left\langle f, \phi_{j, k}\right\rangle\right|^{2} \leq\|f\|_{\infty}^{2}|\operatorname{Sop}(f)|\left[\sum_{k} \int_{2^{-j} \operatorname{Sop}(f)}|\phi(y-k)|^{2} d y\right]
$$

Elegimos $K>0$ tal que

$$
2^{-j} \operatorname{Sop}(f) \bigcap\left[2^{--j} \operatorname{Sop}(f)+K\right]=\emptyset \text {, cuando } k \geq K \text {. }
$$

Entonces en la parte derecha de (3.9), se tiene

$$
\begin{aligned}
\sum_{k} \int_{2^{-, j} \operatorname{Sop}(f)}|\phi(y-k)|^{2} d y & =\sum_{m \in \mathbb{Z}} \sum_{l=0}^{K-1} \int_{2^{-j} \operatorname{Sop}(f)}|\phi(y-m K-l)|^{2} d y \\
& \leq \sum_{l=0}^{K-1} \int_{\bigcup_{m \in \mathbb{Z}}\left(2^{-j} \operatorname{Sop}(f)+l+m K\right)}|\phi(y-l)|^{2} d y
\end{aligned}
$$

y debido a

$$
\left(2^{-j} \operatorname{Sop}(f)+l+m K\right) \bigcap\left(2^{-j} \operatorname{Sop}(f)+l+n K\right)=\emptyset \text {, si } m \neq n ; \forall m, n \in \mathbb{Z}
$$

se sigue que

$$
\sum_{k} \int_{2^{-j} \operatorname{Sop}(f)}|\phi(y-k)|^{2} d y \leq \sum_{l=0}^{K-1} \int_{\mathbb{R}}|\phi(y-l)|^{2} d y .
$$

Luego, desarrollando la sumatoria en el lado derecho de la última desigualdad, obtenemos

$$
\sum_{k} \int_{2^{-j} \operatorname{Sop}(f)}|\phi(y-k)|^{2} d y \leq K\|\phi\|^{2} .
$$

Por consiguiente

$$
\sum_{k}\left|\left\langle f, \phi_{j, k}\right\rangle\right|^{2} \leq\|f\|_{\infty}^{2}|\operatorname{Sop}(f)| K\|\phi\|^{2}
$$

el cual nos permite aseverar que

$$
\sum_{k}\left|\left\langle f, \phi_{j, k}\right\rangle\right|^{2} \text { es convergente } \forall j \in \mathbb{Z} \text {. }
$$

Analogamente se obtendrá que

$$
\sum_{k}\left|\left\langle f, \Psi_{j, k}\right\rangle\right|^{2} \text { es convergente, } \forall j \in \mathbb{Z} \text {. }
$$

(c) Debido a que $\phi=\sum_{n} h_{n} \phi_{-1, n}, \Psi=\sum_{n}(-1)^{n} \bar{h}_{-n+1} \phi_{-1, n}$ y además

$$
\begin{aligned}
\left\langle f, \phi_{j, k}\right\rangle & =\sum_{n} \bar{h}_{n-2 k}\left\langle f, \phi_{j-1, n}\right\rangle \\
\left\langle f, \Psi_{j, k}\right\rangle & =\sum_{n} \bar{g}_{n-2 k}\left\langle f, \phi_{j-1, n}\right\rangle
\end{aligned}
$$

se tiene 


$$
\begin{aligned}
& \left\langle f, \phi_{0, k}\right\rangle=\sum_{n} \bar{h}_{n-2 k}\left\langle f, \phi_{-1, n}\right\rangle \\
& \left\langle f, \Psi_{0, k}\right\rangle=\sum_{n} \bar{g}_{n-2 k}\left\langle f, \phi_{-1, n}\right\rangle
\end{aligned}
$$

Luego

$$
\begin{aligned}
\sum_{k}\left|\left\langle f, \phi_{0, k}\right\rangle\right|^{2} & =\sum_{k} \sum_{m, n}\left[h_{n-2 k} \bar{h}_{m-2 k}\right]\left\langle f, \phi_{-1, n}\right\rangle\left\langle\phi_{-1, m}, f\right\rangle \\
\sum_{k}\left|\left\langle f, \Psi_{0, k}\right\rangle\right|^{2} & =\sum_{k} \sum_{m, n}\left[(-1)^{m+n} \bar{h}_{2 k-n+1} h_{2 k-m+1}\right]\left\langle f, \phi_{-1, n}\right\rangle\left\langle\phi_{-1, m}, f\right\rangle
\end{aligned}
$$

donde un número finito de los $h_{n}$ son no ceros.

(d) Ahora veamos que sucede con las expresiones de (3.10).

Si $n=2 r, m=2 s$ y haciendo $k=s+r-l$, para luego sumar la parte derecha de las expresiones de (3.10), tenemos

$$
\begin{aligned}
& \sum_{k}\left[h_{n-2 k} \bar{h}_{m-2 k}+(-1)^{m+n} \bar{h}_{2 k-n+1} h_{2 k-m+1}\right] \\
= & \sum_{k=k(n, s)}\left[h_{2 l-2 s} \bar{h}_{2 l-2 r}+\bar{h}_{2 s-2 l+1} h_{2 r-2 l+1}\right]
\end{aligned}
$$

y usando (3.6), es decir

$$
\sum_{p} h_{p} \bar{h}_{p-2 k}= \begin{cases}1 ; k=0 \\ 0 ; k \neq 0\end{cases}
$$

obtenemos

$$
\sum_{k}\left[h_{n-2 k} \bar{h}_{m-2 k}+(-1)^{m+n} \bar{h}_{2 k-n+1} h_{2 k-m+1}\right]=\delta_{r, s}=\delta_{n, m}
$$

En forma análoga, para $n=2 r+1, m=2 s+1$ y haciendo $k=s+r-l$, tenemos

$$
\begin{aligned}
& \sum_{k}\left[h_{n-2 k} \bar{h}_{m-2 k}+(-1)^{n+m} \bar{h}_{2 k-n+1} h_{2 k-m+1}\right] \\
= & \sum_{k}\left[h_{2 l-2 s+1} \bar{h}_{2 l-2 r+1}+\bar{h}_{2 s-2 l} h_{2 r-2 l}\right] \\
= & \delta_{r, s}=\delta_{n, m}
\end{aligned}
$$

Asimismo, para $n=2 r, m=2 s+1$ y haciendo $k=s+r-l$, tenemos

$$
\begin{aligned}
& \sum_{k}\left[h_{n-2 k} \bar{h}_{m-2 k}+(-1)^{n+m} \bar{h}_{2 k-n+1} h_{2 k-m+1}\right] \\
= & \sum_{k}\left[h_{2 r-2 k} \bar{h}_{2 s+1-2 k}-\bar{h}_{2 k-2 r+1} h_{2 k-2 s}\right]
\end{aligned}
$$

y como $\sum_{k} h_{p} \bar{h}_{p-2 k}=\delta_{k, 0}$, se tiene

$$
\sum_{k}\left[h_{n-2 k} \bar{h}_{m-2 k}+(-1)^{n+m} \bar{h}_{2 k-n+1} h_{2 k-m+1}\right]=0=\delta_{r, s}=\delta_{n, m}
$$


Luego de (3.11), (3.12) y (3.13), se sigue que

$$
\sum_{k}\left[h_{n-2 k} \bar{h}_{m-2 k}+(-1)^{n+m} \bar{h}_{2 k-n+1} h_{2 k-m+1}\right]=\delta_{n, m} \forall m, n \in \mathbb{Z} .
$$

Por consiguiente

$$
\begin{aligned}
\sum_{k}\left|\left\langle f, \phi_{0, k}\right\rangle\right|^{2}+\sum_{k}\left|\left\langle f, \Psi_{0, k}\right\rangle\right|^{2} & =\sum_{m, n} \delta_{m, n}\left\langle f, \phi_{-1, m}\right\rangle\left\langle\phi_{-1, m}, f\right\rangle \\
& =\sum_{m}\left|\left\langle f, \phi_{-1, m}\right\rangle\right|^{2}
\end{aligned}
$$

Por la propiedad "telescópica", tenemos

$$
\sum_{j=-J+1}^{J} \sum_{k}\left|\left\langle f, \Psi_{j, k}\right\rangle\right|^{2}=\sum_{k}\left|\left\langle f, \phi_{-J, k}\right\rangle\right|^{2}-\sum_{k}\left|\left\langle f, \phi_{J, k}\right\rangle\right|^{2}
$$

(e) Asimismo, se demuestra que para una función fija $f$, continua y de soporte compacto,

$$
\sum_{k}\left|\left\langle f, \phi_{J, k}\right\rangle\right|^{2} \longrightarrow 0, \text { cuando } J \cdot \rightarrow \infty
$$

esto es, $\sum_{k}\left|\left\langle f, \phi_{J, k}\right\rangle\right|^{2} \leq \varepsilon$ para $J$ suficientemente grande y $\varepsilon$ arbitrario.

Similarmente, se prueba que

$$
\sum_{k}\left|\left\langle f, \phi_{-J, k}\right\rangle\right|^{2}=2 \pi \int\left|\widehat{\phi}\left(2^{-J} \xi\right)\right|^{2}|\widehat{f}(\xi)|^{2} d \xi+R
$$

con $|R| \leq C^{\prime \prime} 2^{-J} \leq \varepsilon$, para $J$ suficientemente grande.

Afirmación: $|\widehat{\phi}(\xi)| \leq(2 \pi)^{-\frac{1}{2}}, \forall \xi$.

En efecto, como $\left|m_{0}(\xi)\right| \leq 1, \forall \xi$, entonces es cierto que

$$
\left|m_{0}\left(\frac{\xi}{2^{j}}\right)\right| \leq 1, \forall j=1,2, \ldots k .,
$$

esto nos permite afirmar

$$
\left|\prod_{j=1}^{k} m_{0}\left(\frac{\xi}{2^{j}}\right)\right| \leq 1
$$

y cuando $k \rightarrow \infty$, obtenemos

$$
\left|\prod_{j=1}^{\infty} m_{0}\left(\frac{\xi}{2^{j}}\right)\right| \leq 1
$$

Por otra parte sabemos que $\widehat{\phi}(\xi)=(2 \pi)^{-\frac{1}{2}} \prod_{j=1}^{\infty} m_{0}\left(\frac{\xi}{2^{j}}\right)$, entonces de la desigualdad anterior obtenemos la afirmación deseada.

Ahora, debido a la afirmación anterior y al hecho de que $\widehat{\phi}$ es continua en $\xi=0$, estamos en condiciones de aplicar el Teorema de la Convergencia Dominada de Lebesgue, el cual nos permite aseverar

$$
\sum_{k}\left|\left\langle f, \phi_{-J, k}\right\rangle\right|^{2} \longrightarrow 2 \pi|\widehat{\phi}(0)|^{2} \int|\widehat{f}(\xi)|^{2} d \xi, \text { cuando } J \rightarrow \infty
$$


y como $\widehat{\phi}(0)=(2 \pi)^{-\frac{1}{2}}$ tenemos

$$
\sum_{k}\left|\left\langle f, \phi_{-J, k}\right\rangle\right|^{2} \longrightarrow\|f\|^{2}, \text { cuando } J \rightarrow \infty,
$$

Por consiguiente en (3.14), cuando $J \rightarrow \infty$ obtenemos

$$
\sum_{j=-J+1}^{J} \sum_{k \in \mathbb{Z}}\left|\left\langle f, \Psi_{j, k}\right\rangle\right|^{2} \longrightarrow\|f\|^{2}
$$

Por tanto

$$
\sum_{j, k}\left|\left\langle f, \Psi_{j, k}\right\rangle\right|^{2}=\|f\|^{2}, \forall f \in C_{c}^{\infty}
$$

Finalmente, como la inmersión continua $C_{c}^{\infty} \hookrightarrow L^{2}(\mathbb{R})$ es densa, (3.15) es extendido para todo $f \in L^{2}(\mathbb{R})$. Por consiguiente

$$
\sum_{j, k}\left|\left\langle f, \Psi_{j, k}\right\rangle\right|^{2}=\|f\|^{2}, \forall f \in L^{2}(\mathbb{R}) \Leftrightarrow\left\{\Psi_{j, k}: j, k \in \mathbb{Z}\right\} \text { es b.o.n en } L^{2}(\mathbb{R})
$$

Esto es, $\left\{\Psi_{j, k}: j, k \in \mathbb{Z}\right\}$ es un frame apretado para $L^{2}(\mathbb{R})$.

\section{Otras consideraciones sobre el polinomio trigonométrico $m_{0}$}

Sin considerar condiciones adicionales sobre $m_{0}$, se tiene un frame-apretado con constante frame 1. Esto es,

$$
\sum_{j, k}\left|\left\langle f, \Psi_{j, k}\right\rangle\right|^{2}=\|f\|^{2}, \forall f \in L^{2}(\mathbb{R}) \Leftrightarrow\left\{\Psi_{j, k}: j, k \in \mathbb{Z}\right\} \text { es b.o.n para } L^{2}(\mathbb{R}) .
$$

o equivalentemente

$$
\sum_{j, k}\left|\left\langle f, \Psi_{j, k}\right\rangle\right|^{2}=\|f\|^{2}, \forall f \in L^{2}(\mathbb{R}) \Leftrightarrow \sum_{l}|\widehat{\Psi}(\xi+2 \pi l)|^{2}=(2 \pi)^{-1} .
$$

Además, como $\widehat{\Psi}(\xi)=\exp \left(-\frac{i \xi}{2}\right) \overline{m_{0}\left(\frac{\xi}{2}+\pi\right)} \hat{\phi}\left(\frac{\xi}{2}\right)$ se tiene que

$$
\begin{aligned}
|\widehat{\Psi}(\xi)| & =\left|\exp \left(-\frac{i \xi}{2}\right) \overline{m_{0}\left(\frac{\xi}{2}+\pi\right)} \hat{\phi}\left(\frac{\xi}{2}\right)\right| \\
& =\left|m_{0}\left(\frac{\xi}{2}+\pi\right)\right|\left|\widehat{\phi}\left(\frac{\xi}{2}\right)\right|,
\end{aligned}
$$

entonces

$$
\begin{aligned}
2 \pi \sum_{l \in \mathbb{Z}}|\widehat{\Psi}(\xi+2 \pi l)|^{2} & =2 \pi \sum_{l \in \mathbb{Z}}\left|m_{0}\left(\frac{\xi+2 \pi l}{2}+\pi\right)\right|^{2}\left|\widehat{\phi}\left(\frac{\xi+2 \pi l}{2}\right)\right|^{2} \\
1 & =2 \pi \sum_{l \in \mathbb{Z}}\left|m_{0}\left(\frac{\xi}{2}+\pi l+\pi\right)\right|^{2}\left|\widehat{\phi}\left(\frac{\xi}{2}+\pi l\right)\right|^{2}
\end{aligned}
$$


Ahora, descomponiendo los términos de índices pares e impares de la sumatoria, tenemos

$$
\begin{aligned}
1= & 2 \pi \sum_{l \in \mathbb{Z}}\left|m_{0}\left(\frac{\xi}{2}+2 \pi l+\pi\right)\right|^{2}\left|\widehat{\phi}\left(\frac{\xi}{2}+2 \pi l\right)\right|^{2} \\
& +2 \pi \sum_{l \in \mathbb{Z}}\left|m_{0}\left(\frac{\xi}{2}+2 \pi l+2 \pi\right)\right|^{2}\left|\widehat{\phi}\left(\frac{\xi}{2}+\pi+2 \pi l\right)\right|^{2} .
\end{aligned}
$$

Entonces

$$
\begin{aligned}
1= & 2 \pi \sum_{l \in \mathbb{Z}}\left|m_{0}\left(\frac{\xi}{2}+\pi\right)\right|^{2}\left|\widehat{\phi}\left(\frac{\xi}{2}+2 \pi l\right)\right|^{2} \\
& +2 \pi \sum_{l \in \mathbb{Z}}\left|m_{0}\left(\frac{\xi}{2}\right)\right|^{2}\left|\widehat{\phi}\left(\frac{\xi}{2}+\pi+2 \pi l\right)\right|^{2},
\end{aligned}
$$

el cual puede ser escrito como

$$
\begin{aligned}
1= & \left|m_{0}\left(\frac{\xi}{2}+\pi\right)\right|^{2} 2 \pi \sum_{l \in \mathbb{Z}}\left|\widehat{\phi}\left(\frac{\xi}{2}+2 \pi l\right)\right|^{2} \\
& +\left|m_{0}\left(\frac{\xi}{2}\right)\right|^{2} 2 \pi \sum_{l \in \mathbb{Z}}\left|\widehat{\phi}\left(\frac{\xi}{2}+\pi+2 \pi l\right)\right|^{2}
\end{aligned}
$$

y haciendo $\alpha(\xi)=2 \pi \sum_{l}|\widehat{\phi}(\xi+2 \pi l)|^{2}$ en la última ecuación, obtenemos

$$
\left|m_{0}\left(\frac{\xi}{2}+\pi\right)\right|^{2} \alpha(\xi)+\left|m_{0}\left(\frac{\xi}{2}\right)\right|^{2} \alpha\left(\frac{\xi}{2}+\pi\right)=1
$$

\section{Conclusiones}

5.1. Diversas estrategias han sido desarrolladas, correspondiente a condiciones sobre $m_{0}$, que permiten asegurar que se cumpla

$$
\sum_{l}|\widehat{\phi}(\xi+2 \pi l)|^{2}=(2 \pi)^{-1} \quad \text { ó } \quad \int \phi(x) \bar{\phi}(x-n) d x=\delta_{n, 0}
$$

Una de estas estrategias consiste en probar que las funciones truncamientos $f_{k}$ definidas por:

$$
f_{k}(\xi)=(2 \pi)^{-\frac{1}{2}}\left[\prod_{j=1}^{k} m_{0}\left(2^{-j} \xi\right)\right] \chi_{[-\pi, \pi]}\left(2^{-k} \xi\right)
$$

donde

$$
\chi_{[-\pi, \pi]}\left(2^{-k} \xi\right)=\left\{\begin{array}{l}
1 ;|\xi| \leq 2^{k} \pi \\
0 ; \text { en otro caso }
\end{array}\right.
$$

convergan a $\widehat{\phi}$ en $L^{2}(\mathbb{R})$ y no solo puntualmente en c.t.p.

5.2. Condiciones suficientes sobre el polinomio trigonométrico $m_{0}$ para asegurar está convergencia en $L^{2}(\mathbb{R})$ son dadas por:

(i) $\inf _{|\xi| \leq \frac{\pi}{2}}\left|m_{0}(\xi)\right|>0$ (Mallat 1989). 
(ii) $m_{0}(\xi)=\left(\frac{1+\exp (i \xi)}{2}\right)^{N} \mathcal{L}(\xi)$ con $\sup _{\xi}|\mathcal{L}(\xi)| \leq 2^{N-\frac{1}{2}}$ (Daubechies 1988).

Ninguna de estas condiciones son necesarias, pero ambas cubren muchos ejemplos interesantes.

5.3. Para construir una función $\Psi$ tal que $\left\{\Psi_{j, k}\right\}_{j, k \in \mathbb{Z}}$ sea una base ortonormal de $L^{2}(\mathbb{R})$, seguiremos los siguientes pasos:

(A) Elegir adecuadamente $\phi$ tales que:

(i) $\phi$ y $\widehat{\phi}$ tengan "decaimiento razonable" (regular)

(ii) $\phi$ y $\widehat{\phi}$ deben satisfacer las siguientes características:

$$
\phi(x)=\sum_{n} c_{n} \phi(2 x-n), \sum_{n}\left|c_{n}\right|^{2}<\infty
$$

y

$$
0<\alpha \leq \sum_{l \in \mathbb{Z}}|\widehat{\phi}(\xi+2 \pi l)|^{2} \leq \beta<\infty
$$

(iii) $\int_{\mathbb{R}} \phi(x) d x \neq 0$

(B) Considerar

$$
\widehat{\phi}^{\#}(\xi)=\widehat{\phi}(\xi)\left[2 \pi \sum_{l \in \mathbb{Z}}|\widehat{\phi}(\xi+2 \pi l)|^{2}\right]^{-\frac{1}{2}}
$$

el cual ejecuta la llamada "ortonormalización trucada".

(C) Finalmente, se tiene

$$
\widehat{\Psi}(\xi)=\exp \left(\frac{i \xi}{2}\right) m_{0}^{\#}\left(\frac{\frac{\xi}{2}}{2}+\pi\right) \widehat{\phi}^{\#}\left(\frac{\xi}{2}\right)
$$

donde

$$
m_{0}^{\#}(\xi)=m_{0}(\xi)\left[\sum_{l \in \mathbb{Z}}|\widehat{\phi}(2 \xi+2 \pi l)|^{2}\right]^{-\frac{1}{2}}\left[\sum_{l \in \mathbb{Z}}|\widehat{\phi}(\xi+2 \pi l)|^{2}\right]^{\frac{1}{2}}
$$

o equivalentemente

$$
\Psi(x)=\sum_{n}(-1)^{n} h_{1-n}^{\#} \phi^{\#}(x-n), \quad \text { con } m_{0}^{\#}(\xi)=2^{-\frac{1}{2}} \sum_{n} h_{n}^{\#} \exp (-i n \xi)
$$

Luego, construir ejemplos de bases ortonormales en la $\Psi$ tiene soporte compacto es más factible comenzar de un polinomio trigonométrico $m_{0}$, más que de la función escala $\phi$ ó de los espacios $V_{j}$. 


\section{REFERENCIAS BIBLIOGRÁFICAS}

[1] BEYLKIN G., COIFMAN R., ROKHLIN V., Fast Wavelet Transforms and numerical algorithms, Comm. Pure and Appl. Math., 44, (1991), pp.141-183.

[2] BURKE-HUBBAR, B., Ondes et Ondelettes, pour la science, París, (1995).

[3] CHUI, C., An Introduction to Wavelets. Academic Press, Boston, (1992).

[4] CHUI, C., Wavelets: a Tutorial in Theory and Applications. Academic Press, Boston, (1992).

[5] COHEN A., DAUBECHIES I., \& Vial, P., Wavelets on the interval and fast wavelet transform, Journal of Applied and Computational Harmonic Analysis 1, (1993), pp. 54-81.

[6] DAUBECHIES I., Ten lectures on Wavelets, CBMS-NSF Regional Conference Series in Applied Mathematics, SIAM Publ. Philadelphia, (1992).

[7] DAUBECHIES I., Orthonormal bases of Compactly Supported Wavelets, Comm. Pure and Appl. Math. 41, (1988), pp. 909-996. 